\title{
TOTALLY SYMMETRIC SURFACES OF CONSTANT MEAN CURVATURE IN HYPERBOLIC 3-SPACE
}

\author{
SHIMPEI KOBAYASHI
}

(Received 1 December 2009)

\begin{abstract}
We detail a construction of totally symmetric surfaces of constant mean curvature $0 \leq H<1$ in hyperbolic 3-space of sectional curvature -1 via the generalized Weierstrass type representation.

2000 Mathematics subject classification: primary 53A10, 58D10; secondary 53C42.

Keywords and phrases: constant mean curvature, hyperbolic 3-space, generalized Weierstrass type representation.
\end{abstract}

\section{Introduction}

It has been recognized that the generalized Weierstrass type representation for constant mean curvature (CMC for short) surfaces in $\boldsymbol{R}^{3}$ is a powerful tool for producing CMC surfaces with nontrivial topologies $[1,3,5,7,11]$. The heart of the construction is a second-order linear ordinary differential equation (ODE) of one complex variable, which is derived from a CMC surface, with an additional 'loop parameter'. More precisely, for the construction of CMC surfaces with nontrivial topologies the monodromy group of the complex linear ODE needs to be a unitary loop subgroup, which will be referred to as the 'unitarizability problem'. The unitarizability problem is an essential part of the construction of CMC surfaces via the generalized Weierstrass type representation.

The Gauss hypergeometric equation is a second-order linear ODE whose solutions have three regular singularities on the extended complex plane $\hat{\boldsymbol{C}}=\boldsymbol{C} \cup\{\infty\}$, and its monodromy group is completely understood. Thus it was natural to expect that one can construct corresponding CMC surfaces which are well defined on the thrice punctured extended complex plane $\hat{\boldsymbol{C}}$. It turns out that such CMC surfaces exist and are called CMC trinoids $[5,11]$. The asymptotic behaviours of the CMC trinoid around the punctures, which are referred to as the ends, had been expected to be geometrically 'nice', since those ends correspond to regular singularities of the Gauss hypergeometric equation. In fact, it is shown that each end of the CMC trinoids is

This work was partially supported by Kakenhi 20740045.

(C) 2010 Australian Mathematical Publishing Association Inc. 0004-9727/2010 \$16.00 
asymptotic to half of a CMC surface of revolution [5, 8]. Moreover, the generalized Weierstrass type representation can be generalized for CMC surfaces in $S^{3}$, and in $\boldsymbol{H}^{3}$ with mean curvature $H>1$, [11]. Similar to the $\boldsymbol{R}^{3}$ case, there also exist CMC trinoids in $S^{3}$, and in $\boldsymbol{H}^{3}$ with mean curvature $H>1$, [11].

Recently, in [2, 9], the generalized Weierstrass type representation for CMC surfaces in $\boldsymbol{H}^{3}$ with mean curvature $0 \leq H<1$ has been established. It is shown that there also exist CMC surfaces using the Gauss hypergeometric equation [2]. The difference between CMC trinoids in $\boldsymbol{R}^{3}$ and CMC surfaces in $\boldsymbol{H}^{3}$ via the Gauss hypergeometric equation is the existence of singular curves. In fact, CMC trinoids in $\boldsymbol{R}^{3}$ do not have any singularity except the three regular singularities, which correspond to the ends of CMC trinoids, of the Gauss hypergeometric equation. In contrast, those CMC surfaces in $\boldsymbol{H}^{3}$ have singular curves, where the surfaces tends to infinity, in addition to three regular singularities. Moreover, until now, we have only been able to show the existence of the particular type of such surfaces such that each end is the same type. Therefore, it is natural to call such a surface the totally symmetric surface.

This paper gives a detailed computation for the construction of totally symmetric surfaces in $\boldsymbol{H}^{3}$ with mean curvature $0 \leq H<1$, [2]. Similar to the $\boldsymbol{R}^{3}$ case, for the construction of CMC surfaces in $\boldsymbol{H}^{3}$ with mean curvature $0 \leq H<1$, a monodromy group of the complex linear ODE needs to be a particular real loop subgroup, which is referred to as the 'realizability problem'. The method for the realizability problem is similar to the method for the unitarizability problem of CMC trinoids in $\boldsymbol{R}^{3}$; however, the resulting conditions are different [4].

For simplicity of presentation, we assume the following.

(1) The eigenvalues of the monodromy matrices are even in the loop parameter.

(2) The loop parameter is in $S^{1}$.

Assumptions (1) and (2) are closely related. If the eigenvalue is odd in the loop parameter, then the loop parameter needs to be chosen in $S^{r}$, the circle of radius $0<r<1$, to solve the realizability problem. However, for most cases, eigenvalues of monodromy matrices are even with respect to the loop parameter, so these are not severe restrictions.

\section{Preliminaries}

In this section we give basic notation and results.

2.1. Basic definitions. Let $G^{C}$ be a semisimple complex Lie group. The twisted loop group is defined as

$$
\Lambda G_{\sigma}^{C}=\left\{g: S^{1} \rightarrow G^{C} \mid g \text { is continuous and } g(-\lambda)=\sigma g(\lambda)\right\},
$$

where $\sigma$ is an involution. More strictly, we assume that the coefficients of all $g \in \Lambda G_{\sigma}^{C}$ are in the Wiener algebra

$$
\mathcal{W}=\left\{f(\lambda)=\sum_{n \in \boldsymbol{Z}} f_{n} \lambda^{n}: S^{1} \rightarrow \boldsymbol{C} ; \sum_{n \in \boldsymbol{Z}}\left|f_{n}\right|<\infty\right\} .
$$


The Wiener algebra $\mathcal{W}$ is a Banach algebra relative to the norm $\|f\|=\sum\left|f_{n}\right|$, and $\mathcal{W}$ consists of continuous functions. Thus $\Lambda G_{\sigma}^{C}$ is a Banach Lie group. We denote its Lie algebra by $\Lambda \mathfrak{g}_{\sigma}^{C}$. Define $\mathcal{W}^{+}$and $\mathcal{W}^{-}$to be the subalgebras of $\mathcal{W}$ which consists of loops whose Fourier expansions have only nonnegative, respectively nonpositive, powers of $\lambda$.

We will need to consider two subgroups of $\Lambda G_{\sigma}^{C}$, the twisted plus loop group and the minus loop group. Let $D=\{\lambda \in C|| \lambda \mid<1\}$ be a radius-one disk, and let $E=\{\lambda \in C|1<| \lambda \mid\} \cup\{\infty\}$ the exterior of the radius-one disk. We denote the closure of $D$ and $E$ by $\bar{D}^{\text {cl }}$ and $\bar{E}$, respectively. Moreover, let $B$ be a subgroup of $G^{C}$. Then

$$
\begin{gathered}
\Lambda_{B}^{+} G_{\sigma}^{C}=\left\{g \in \Lambda G_{\sigma}^{C} \mid g \text { can be holomorphically extended to } D\right. \\
\text { and takes values in } B \text { at } \lambda=0\}, \\
\Lambda_{B}^{-} G_{\sigma}^{C}=\left\{g \in \Lambda G_{\sigma}^{C} \mid g \text { can be holomorphically extended to } E\right. \\
\text { and takes values in } B \text { at } \lambda=\infty\} .
\end{gathered}
$$

If $B=\{$ id $\}$, we write the subscript $*$ instead of $B$, and if $B=G^{C}$ we abbreviate $\Lambda_{B}^{+} G_{\sigma}^{C}$ and $\Lambda_{B}^{-} G_{\sigma}^{C}$ by $\Lambda^{+} G_{\sigma}^{C}$ and $\Lambda^{-} G_{\sigma}^{C}$, respectively.

2.2. Loop groups factorizations. From now on, we consider only $\Lambda \mathrm{SL}_{2} \boldsymbol{C}_{\sigma}$ as $\Lambda G_{\sigma}^{\boldsymbol{C}}$, where the involution $\sigma$ is given by $\operatorname{Ad}\left(\begin{array}{cc}1 & 0 \\ 0 & -1\end{array}\right)$. We introduce some useful notation. For $f \in \mathcal{W}$, the $\sharp$ and b operations are defined as follows:

$$
f^{\sharp}=\overline{f(i / \bar{\lambda})}^{-1} \text { and } f^{b}=\overline{f(i / \bar{\lambda})} \text {. }
$$

For $g \in \Lambda G_{\sigma}^{C}$, the $\sharp$ operation is defined as follows:

$$
g^{\sharp}=\operatorname{Ad}(\mathcal{R}) \overline{g(i / \bar{\lambda})}^{t-1} \quad \text { with } \mathcal{R}=\left(\begin{array}{cc}
1 / \sqrt{i} & 0 \\
0 & \sqrt{i}
\end{array}\right) .
$$

The subgroup $\Lambda G_{\sigma}$ of $\Lambda G_{\sigma}^{C}$ is defined as

$$
\Lambda G_{\sigma}=\left\{g \in \Lambda G_{\sigma}^{C} \mid g^{\sharp}=g\right\},
$$

which is a real form of $\Lambda G_{\sigma}^{C}$. It is easy to check that, for $g=\left(g_{i j}\right) \in \Lambda G_{\sigma}^{C}$, the condition $g^{\sharp}=g$ can be computed via the entries of $g$ as $g_{11}=g_{22}^{b}$ and $g_{21}=-i g_{12}^{b}$.

REMARK 2.1. The loop subgroup $\Lambda G_{\sigma}$ is not defined by any finite-dimensional real form $G$ of $G^{C}$, that is,

$$
\Lambda G_{\sigma} \neq\left\{g: S^{1} \rightarrow G \mid g(-\lambda)=\sigma g(\lambda)\right\} .
$$

The real form naturally induces an Iwasawa decomposition of $\Lambda G_{\sigma}^{C}$.

THEOREM 2.2 [2]. $\Lambda G_{\sigma}^{\boldsymbol{C}}$ can be factorized as

$$
\Lambda G_{\sigma}^{C}=\bigcup_{n=0}^{\infty} \Lambda G_{\sigma} \omega_{n} \Lambda^{+} G_{\sigma}^{C},
$$


where $\omega_{n}$ is the middle term of the form $\omega_{n}=\left(\begin{array}{cc}\lambda^{n} & 0 \\ 0 & \lambda^{-n}\end{array}\right)$ for $n=2 k$ and $\omega_{n}=\left(\begin{array}{cc}0 & \lambda^{-n} \\ -\lambda^{n} & 0\end{array}\right)$ for $n=2 k+1$. Moreover, $\Lambda G_{\sigma} \Lambda^{+} G_{\sigma}^{C} \cup \Lambda G_{\sigma} \omega_{1} \Lambda^{+} G_{\sigma}^{C}$ is the open dense subset of $\Lambda G_{\sigma}^{C}$, which is called the big cell.

We quote the Birkhoff decomposition for functions, which will be used later.

LEMMA 2.3 [6]. $h \in \mathcal{W}$ can be factorized as

$$
h(\lambda)=c \lambda^{m} h_{+}(\lambda) h_{-}(\lambda),
$$

where $h_{ \pm} \in \mathcal{W}^{ \pm}$are nonvanishing holomorphic functions on $D$ and $E$, respectively, with $h_{+}(0)=h_{-}(\infty)=1, c$ is a nonzero constant and $m \in \boldsymbol{Z}$.

2.3. Generalized Weierstrass type representation. In this subsection, we briefly explain the generalized Weierstrass type representation for CMC surfaces in $\boldsymbol{H}^{3}$ with mean curvature $0 \leq H<1$. We refer the reader to [2] for more details.

Step 1. Let $\eta$ be a holomorphic potential of the form

$$
\eta=\sum_{j=-1}^{\infty} \eta_{j} \lambda^{j}
$$

where $\eta_{\text {odd }}$ (respectively $\eta_{\text {even }}$ ) is an off-diagonal (respectively diagonal) $\mathfrak{s l}_{2} \boldsymbol{C}$-valued holomorphic 1-form defined on $\Sigma \subset \boldsymbol{C}$, and the upper right entry of $\eta_{-1}$ does not have a zero on $\Sigma$.

Step 2. Solve the ODE

$$
d C=C \eta
$$

for some initial condition $C\left(z_{*}\right)$.

Step 3. Perform the Iwasawa decomposition in Theorem 2.2 on the big cell:

$$
C=F V_{+}, \quad F \in \Lambda G_{\sigma} \quad \text { and } \quad V_{+} \in \Lambda^{+} G_{\sigma}^{C}
$$

or

where $\omega_{1}=\left(\begin{array}{cc}0 & \lambda^{-1} \\ -\lambda & 0\end{array}\right)$.

$$
C=F \omega_{1} V_{+}, \quad F \in \Lambda G_{\sigma} \quad \text { and } \quad V_{+} \in \Lambda^{+} G_{\sigma}^{C},
$$

Step 4. Plug $F$ or $F \omega_{1}$ into the Sym formula

$$
\psi=\left.F\left(\begin{array}{cc}
e^{-q / 2} & 0 \\
0 & e^{q / 2}
\end{array}\right) F^{*}\right|_{\lambda=e^{-q / 2}}
$$

or

$$
\psi=-\left.F \omega_{1}\left(\begin{array}{cc}
e^{-q / 2} & 0 \\
0 & e^{q / 2}
\end{array}\right) \omega_{1}^{*} F^{*}\right|_{\lambda=e^{-q / 2}},
$$

respectively, where $X^{*}$ denotes $\bar{X}^{t}$. Then $\psi$ is a constant mean curvature immersion in $\boldsymbol{H}^{3}$ with mean curvature $H=\tanh q$.

REMARK 2.4. As explained in [2, Section 9.4], the resulting immersion $\psi$ by the Sym formula (2.2) for $F$ can be extended meromorphically to $\boldsymbol{C}^{2}$. Then restricting $\psi$ again 
to the other component of the big cell, the Sym formula $\psi$ with $F \omega_{1}$ is obtained as in (2.3), and the resulting immersion can be shown in $\boldsymbol{H}^{3}$ of the lower half.

2.4. Monodromy problem. It is known that, for a CMC surface defined on a noncompact Riemann surface $\Sigma$, there exists a holomorphic potential $\eta$ which is well defined on $\Sigma$, [2, Section 8.2]. Thus constructing a noncompact CMC surface, one can chose a holomorphic potential $\eta$ which is well defined on a noncompact Riemann surface $\Sigma$. It is well known that solutions of the ODE $d C=C \eta$ are not well defined on $\Sigma$, that is, solutions have monodromy matrices. Then the following lemma holds.

LEMMA 2.5. Let $\gamma$ be an element of $\pi_{1}(\Sigma)$, the fundamental group of $\Sigma$, and $M=\left(\gamma^{*} C\right) C^{-1}$ the monodromy matrix. Assume that:

(1) $M \in \Lambda G_{\sigma}$;

(2) $\left.M\right|_{\lambda=e^{-q / 2}}=\mathrm{id}$ or $-\mathrm{id}$.

Then the resulting immersion $\psi$ obtained by the generalized Weierstrass type representation has $\gamma$ as an element of the fundamental group of $\psi$.

PROOF. From the first condition, one can easily see that the Iwasawa decomposition for $\gamma^{*} C$ can be computed as

$$
\gamma^{*} C=(M F) \omega_{n} V_{+}, \quad M F \in \Lambda G_{\sigma} \quad \text { and } \quad V_{+} \in \Lambda^{+} G_{\sigma}^{C},
$$

where $C=F \omega_{n} V_{+}$is the Iwasawa decomposition of $C$. Thus $\gamma^{*} F=M F$ and $\gamma^{*} \psi=\left.M \psi M^{*}\right|_{\lambda=e^{-q / 2}}$. Therefore, the second condition implies that $\gamma^{*} \psi=\psi$.

2.5. Eigenvalues of surfaces of revolution. Set

$$
\eta=\frac{1}{z} A d z \quad \text { with } A=\left(\begin{array}{cc}
c & a \lambda^{-1}+b \lambda \\
-a \lambda+b \lambda^{-1} & -c
\end{array}\right),
$$

where $a, b, c \in \boldsymbol{R}$ and $c^{2}-a^{2}+b^{2}+a b\left(e^{q}-e^{-q}\right)=1 / 4$. Then the ODE $d C=C \eta$ with the initial condition $C\left(z_{*}=1\right)=$ id can be solved explicitly as $C=\exp (\log z A)$. The monodromy matrix around $z=0$ is $M=\exp (2 \pi i A) \in \Lambda G_{\sigma}$. Then a straightforward computation shows that the eigenvalues of $M$ are

$$
\left.\mu_{ \pm}=\exp ( \pm 2 \pi i \sqrt{-\operatorname{det} A})=\exp \left( \pm 2 \pi i \sqrt{c^{2}-a^{2}+b^{2}+a b\left(\lambda^{-2}-\lambda^{2}\right.}\right)\right) .
$$

Thus $\left.\mu_{ \pm}\right|_{\lambda=e^{-q / 2}}=-1$ and $\left.M\right|_{\lambda=e^{-q / 2}}=-\mathrm{id}$. From Lemma 2.5 , the resulting surface $\psi$ closes up.

Then the following lemma holds.

LeMma 2.6. Let $\mu_{ \pm}$be eigenvalues defined in (2.4). Assume that $c^{2}-a^{2}+b^{2} \neq 0$. Then $\mu_{ \pm}$satisfy

$$
\mu_{ \pm}^{\sharp}=\mu_{ \pm} \quad \text { on } S^{1} \text {. }
$$


ProOF. Since the $c^{2}-a^{2}+b^{2}<0$ case is similar, we consider only the $c^{2}-a^{2}+$ $b^{2}>0$ case. A direct computation shows that

$$
\begin{aligned}
-\operatorname{det} A & =c^{2}-a^{2}+b^{2}+a b\left(\lambda^{-2}-\lambda^{2}\right) \\
& =p+2 i a b \sin \theta,
\end{aligned}
$$

where $p=c^{2}-a^{2}+b^{2}>0$ and $\lambda^{-2}=e^{i \theta} \in S^{1}$. Since $-\operatorname{det} A$ is never zero by the assumption, $\sqrt{-\operatorname{det} A}$ is well defined on $S^{1}$. Set $X=\sqrt{-\operatorname{det} A}$. The positivity of $p$ implies that $X^{2}$ takes values in the right half space $\check{\boldsymbol{H}}$ of $\boldsymbol{C}$, and $X$ also takes values in $\check{\boldsymbol{H}}$. It is easy to check that $X^{2 b}=X^{2}$ on $S^{1}$, thus $X=X^{\mathrm{b}}$ or $X=-X^{\mathrm{b}}$. Since $X^{\mathrm{b}}$ takes values in $\check{\boldsymbol{H}}$, however, we conclude that $X^{\mathrm{b}}=X$. Therefore,

$$
\mu_{ \pm}^{\#}=\exp \left( \pm 2 \pi i X^{b}\right)=\exp ( \pm 2 \pi i X)=\mu_{ \pm} .
$$

This completes the proof.

REMARK 2.7. Lemma 2.6 does not hold for the $c^{2}-a^{2}+b^{2}=0$ case, since det $A$ has a zero on $S^{1}$. A similar case is the round cylinder, as a CMC surface in $\boldsymbol{R}^{3}$, [4].

\section{Realizability of loops}

\subsection{Realizability of one loop.}

Definition 3.1. Let $L$ be an element in $\Lambda G_{\sigma}^{C}$. Then $L$ is realizable by $S$ or dressing, if $S L S^{-1} \in \Lambda G_{\sigma}$ for some $S \in \Lambda G_{\sigma}^{C}$, that is, $\left(S L S^{-1}\right)^{\sharp}=S L S^{-1}$.

THEOREM 3.2. Let $L \in \Lambda G_{\sigma}^{\boldsymbol{C}} \backslash\{ \pm \mathrm{id}\}$ be a holomorphic loop around $S^{1}$ such that $T L T^{-1}=\left(\begin{array}{cc}v & 0 \\ 0 & v^{-1}\end{array}\right) \in \Lambda G_{\sigma}$ for some $T \in \Lambda G_{\sigma}^{C}$. Moreover, assume that $L$ is realizable by $S \in \Lambda G_{\sigma}^{C}$. Then $S$ has the form $S=U \omega_{n} G_{+} T$, where $U \in \Lambda G_{\sigma}, \omega_{n}=\left(\begin{array}{cc}\lambda^{n} & 0 \\ 0 & \lambda^{-n}\end{array}\right)$ for $n=2 k$ or $\omega_{n}=\left(\begin{array}{cc}0 & \lambda^{-n} \\ -\lambda^{n} & 0\end{array}\right)$ for $n=2 k+1$ and a diagonal $G_{+} \in \Lambda^{+} G_{\sigma}^{C}$.

PROOF. First, we note that the assumptions imply that $v-v^{-1}$ equals zero at finitely many points at most and $v^{b}=v^{-1}$. Let $S T^{-1}=U \omega_{n} G_{+}$be the Iwasawa decomposition of $S T^{-1}$, that is, $U \in \Lambda G_{\sigma}, \omega_{n}=\left(\begin{array}{cc}\lambda^{n} & 0 \\ 0 & \lambda^{-n}\end{array}\right)$ for $n=2 k$ or $\omega_{n}=\left(\begin{array}{cc}0 & \lambda^{-n} \\ -\lambda^{n} & 0\end{array}\right)$ for $n=2 k+1$ and $G_{+} \in \Lambda^{+} G_{\sigma}^{C}$. Set $G_{+}=\left(\begin{array}{ll}a & b \\ c & d\end{array}\right)$. We show that $b=c=0$. A straightforward computation shows that

$$
\begin{aligned}
U^{-1} S L S^{-1} U & =\omega_{n} G_{+}\left(\begin{array}{cc}
v & 0 \\
0 & v^{-1}
\end{array}\right) G_{+}^{-1} \omega_{n}^{-1} \\
& =\omega_{n}\left(\begin{array}{cc}
v+b c\left(v-v^{-1}\right) & a b\left(v^{-1}-v\right) \\
c d\left(v-v^{-1}\right) & v^{-1}+b c\left(v^{-1}-v\right)
\end{array}\right) \omega_{n}^{-1} .
\end{aligned}
$$

We recall that for a loop $X=\left(x_{i j}\right) \in \Lambda G_{\sigma}^{C}, X \in \Lambda G_{\sigma} \Leftrightarrow x_{22}=x_{11}^{b}$ and $x_{21}=-i x_{12}^{b}$, and $\omega_{n}^{\sharp}= \pm \omega_{n}$. Thus $U^{-1} S L S^{-1} U \in \Lambda G_{\sigma}$ is equivalent to

$$
b c=(b c)^{b}, \quad c d=-i(a b)^{b} \quad \text { on } S^{1} .
$$


Since $x^{b}=\overline{x(i / \bar{\lambda})}$ and $b, c$ are holomorphic on $D, b c$ has a holomorphic extension to $E$. Therefore $b c$ is holomorphic on $\hat{\boldsymbol{C}}$ except $S^{1}$. However, $b c$ is continuous on $S^{1}$, thus $b c$ can be extended holomorphically to $\hat{\boldsymbol{C}}$ [10, p. 289]. Therefore $b c$ is constant. $\left.b c\right|_{\lambda=0}=0$ implies that $b=0$ or $c=0$. Also, $a d-b c=1$ implies that $a d=1$. Therefore the second equation in (3.1) implies that $b=c=0$. This completes the proof.

Moreover, we have the following lemma.

LEMMA 3.3. Let $L=\left(l_{i j}\right) \in \Lambda G_{\sigma}^{C}$ be holomorphic around $S^{1}$. Assume that $l_{12}=0$ and $l_{22}=l_{11}^{b}$. Then the following statements hold.

(1) If $l_{11} \equiv l_{22}$, then $L$ is realizable via dressing if and only if $L= \pm \mathrm{id}$.

(2) If $l_{11} \not \equiv l_{22}$, then $L$ is realizable via dressing if $l_{21} /\left(l_{22}-l_{11}\right)$ does not have any pole on $S^{1}$.

PROOF. (1) From $l_{11} \equiv l_{22}$ and $l_{22}=l_{11}^{-1}$, we have $l_{11}=l_{22}= \pm 1$. Thus the claim follows.

(2) We set

$$
T=\left(\begin{array}{cc}
1 & 0 \\
l_{21} /\left(l_{22}-l_{11}\right) & 1
\end{array}\right) .
$$

Since $l_{21} /\left(l_{22}-l_{11}\right)$ does not have any pole on $S^{1}$, then $T \in \Lambda G_{\sigma}^{C}$. It is easy to see that $T L T^{-1}=\left(\begin{array}{cc}l_{11} & 0 \\ 0 & l_{22}\end{array}\right) \in \Lambda G_{\sigma}$, since $l_{11}$ and $l_{22}$ are even in $\lambda$ and $l_{22}=l_{11}^{b}$.

REMARK 3.4.

(1) In [4, Proposition 2.32], it is shown that the condition (2) in Lemma 3.3 also gives the necessary condition of the unitarizability of the lower triangular loop. To prove this, the reality of $b c$ of $G_{+}$as in Theorem 3.2 is important [4, proof of Theorem 2.16(iii)]. Since $b c$ of $G_{+}$in Theorem 3.2 is not real for our case, it is not clear that it also gives the necessary condition.

(2) If $L$ is the upper triangular loop, that is, $l_{21}=0$ in Lemma 3.3, then the statements also hold under the replacement of $l_{21} /\left(l_{22}-l_{11}\right)$ by $-l_{12} /\left(l_{22}-l_{11}\right)$.

\subsection{Simultaneous realization.}

LEMMA 3.5. Let $h$ be a finite, nonvanishing function on $S^{1}$. Moreover, assume that $h=h^{b}$ on $S^{1}$. Then $h= \pm k_{+}^{n}\left(k_{+}^{n}\right)^{b}$ for some $k_{+} \in \mathcal{W}^{+}$which is finite, non-vanishing on $S^{1}$ and nonvanishing holomorphic on D. Moreover, if $h$ is even, then $k_{+}$is even.

PROOF. Let $h=c \lambda^{m} h_{+} h_{-}$be the Birkhoff decomposition on $S^{1}$ in Lemma 2.3, where $c \in \boldsymbol{C} \backslash\{0\}, m \in \boldsymbol{Z}$, and $h_{ \pm} \in \mathcal{W}^{ \pm}$are nonvanishing holomorphic on $D$ and $E$, respectively. Since $h=h^{\mathrm{b}}$, we have $m=0, c \in \boldsymbol{R} \backslash\{0\}$ and $h_{-}=h_{+}^{\mathrm{b}}$. Since $h_{+}$never vanishes on $\bar{D}^{\text {cl }}$, we can take $k_{+}=\left(c^{1 / 2} h_{+}\right)^{1 / n}$ if $c>0$ and $k_{+}=\left((-c)^{1 / 2} h_{+}\right)^{1 / n}$ if $c<0$. Thus, $h=k_{+}^{n}\left(k_{+}^{n}\right)^{\mathrm{b}}$ if $c>0$ and $h=-k_{+}^{n}\left(k_{+}^{n}\right)^{\mathrm{b}}$ if $c<0$. Uniqueness of the Birkhoff decomposition implies that if $h$ is even, then $k_{+}$is also even. This completes the proof. 
THEOREM 3.6. Let $L_{j} \in \Lambda G_{\sigma}^{C} \backslash\{ \pm \mathrm{id}\},(j=1,2)$, be holomorphic loops around $S^{1}$ such that

$$
T_{j} L_{j} T_{j}^{-1}=\left(\begin{array}{cc}
v_{j} & 0 \\
0 & v_{j}^{-1}
\end{array}\right) \in \Lambda G_{\sigma}
$$

for some $T_{j} \in \Lambda G_{\sigma}^{C},(j=1,2)$. Set

$$
T_{1} T_{2}^{-1}=\left(\begin{array}{ll}
a & b \\
c & d
\end{array}\right) .
$$

Assume that none of $a, b, c, d$ is identically zero on $S^{1}$. Then $L_{1}$ and $L_{2}$ are simultaneously realizable via dressing if and only if

$$
\frac{d}{a^{b}}= \pm f f^{b}, \quad i \frac{c}{b^{b}}= \pm g g^{b}
$$

for some $f, g \in \mathcal{W}^{+}$such that $f, g$ are finite, nonvanishing on $S^{1}$ and nonvanishing holomorphic on $D$, the signs in the left-and right-hand side of the above equation must be in the same order.

ProOF. $(\Rightarrow)$ Let $S$ be a loop such that $S L_{j} S^{-1} \in \Lambda G_{\sigma}(j=1,2)$. Then from Theorem 3.2, $S$ can be decomposed as

$$
S=U_{1} \omega_{1, n} G_{1} T_{1}=U_{2} \omega_{2, n} G_{2} T_{2},
$$

where

$$
U_{j} \in \Lambda G_{\sigma}, \quad G_{j}=\left(\begin{array}{cc}
k_{j} & 0 \\
0 & k_{j}^{-1}
\end{array}\right) \in \Lambda^{+} G_{\sigma}^{C}
$$

and $\omega_{j, n}=\left(\begin{array}{cc}\lambda^{n} & 0 \\ 0 & \lambda^{-n}\end{array}\right)$ for $n=2 k$ or $\omega_{j, n}=\left(\begin{array}{cc}0 & \lambda^{-n} \\ -\lambda^{n} & 0\end{array}\right)$ for $n=2 k+1(j=1,2)$. Since

$$
U_{1}^{-1} U_{2}=\omega_{1, n} G_{1} T_{1} T_{2}^{-1} G_{2}^{-1} \omega_{2, n}^{-1} \in \Lambda G_{\sigma}
$$

and $\omega_{j, n}^{\sharp}= \pm \omega_{j, n}$,

$$
d k_{1}^{-1} k_{2}= \pm\left(a k_{1} k_{2}^{-1}\right)^{b}, \quad c k_{1}^{-1} k_{2}^{-1}=\mp i\left(b k_{1} k_{2}\right)^{b},
$$

where the signs in the left- and right-hand side of the above equation must be in the same order. This is equivalent to

$$
\frac{d}{a^{b}}= \pm \frac{k_{1} k_{1}^{b}}{k_{2} k_{2}^{b}}, \quad i \frac{c}{b^{b}}= \pm k_{1} k_{1}^{b} k_{2} k_{2}^{b} .
$$

Therefore $d / a^{b}$ and $i c / b^{b}$ have the form $d / a^{b}= \pm f f^{b}$ and $i c / b^{b}= \pm g g^{b}$, where $f=k_{1} / k_{2}, g=k_{1} k_{2} \in \mathcal{W}^{+}$. From (3.2), $f$ and $g$ are finite, nonvanishing on $S^{1}$ and nonvanishing holomorphic on $D$.

$(\Leftarrow)$ Conversely, assume that $d / a^{b}= \pm f f^{b}$ and $i c / b^{b}= \pm g g^{b}$, where $f, g \in \mathcal{W}^{+}$ are finite, nonvanishing on $S^{1}$ and nonvanishing holomorphic on $D$. First, we can rephrase $f f^{b}$ and $g g^{b}$ as

$$
f f^{b}= \pm \frac{1}{m} \hat{f} \hat{f}^{b}, \quad g g^{b}= \pm n \hat{g} \hat{g}^{b}
$$


such that

$$
\left.\hat{f}\right|_{\lambda=0}=\left.\hat{f}^{b}\right|_{\lambda=\infty}=\left.\hat{g}\right|_{\lambda=0}=\left.\hat{g}^{b}\right|_{\lambda=\infty}=1,
$$

where $n$ and $m$ are positive constants. Since $\hat{f}, \hat{g}$ are nonvanishing functions on $\bar{D}$, we have $\hat{f}=\exp h_{+}, \hat{g}=\exp l_{+}$for some $h_{+}, l_{+} \in \mathcal{W}^{+}$. Thus the square roots of $\hat{f}$ and $\hat{g}$ are well defined. Set

$$
k_{1}=\left(\frac{n}{m}\right)^{1 / 4}(\hat{f} \hat{g})^{1 / 2}, \quad k_{2}=(n m)^{1 / 4}\left(\frac{\hat{g}}{\hat{f}}\right)^{1 / 2} .
$$

It is easy to see that $k_{1}, k_{2}$ are finite, nonvanishing on $S^{1}$ and nonvanishing holomorphic on $D$. Moreover, $\left.k_{1}\right|_{\lambda=0}>0,\left.k_{2}\right|_{\lambda=0}>0$. Set

$$
K_{j}=\left(\begin{array}{cc}
k_{j} & 0 \\
0 & k_{j}^{-1}
\end{array}\right) \quad(j=1,2)
$$

and $\omega=\mathrm{id}$ for $d / a^{b}=f f^{\mathrm{b}}$ and $i c / b^{\mathrm{b}}=g g^{\mathrm{b}}$, or $\omega=\left(\begin{array}{cc}0 & \lambda^{-1} \\ -\lambda & 0\end{array}\right)$ for $d / a^{b}=-f f^{\mathrm{b}}$ and $i c / b^{b}=-g g^{b}$. Then a straightforward computation shows that

$$
\omega K_{1} T_{1} T_{2}^{-1} K_{2}^{-1}=\omega\left(\begin{array}{cc}
a k_{1} k_{2}^{-1} & b k_{1} k_{2} \\
c k_{1}^{-1} k_{2}^{-1} & d k_{1}^{-1} k_{2}
\end{array}\right) .
$$

It is easy to check that $d k_{1}^{-1} k_{2}= \pm\left(a k_{1} k_{2}^{-1}\right)^{b}$ and $c k_{1}^{-1} k_{2}^{-1}=\mp i\left(b k_{1} k_{2}\right)^{b}$ hold. Since $\left(k_{1} k_{1}^{b}\right)^{2}=i c d /(a b)^{b}$ and $\left(k_{2} k_{2}^{b}\right)^{2}=i(a c)^{b} /(b d)$ on $S^{1}, k_{1}$ and $k_{2}$ are even in $\lambda$ (see Lemma 3.5). Thus $G_{j}=K_{j} \in \Lambda^{+} G_{\sigma}^{C}$. By using $\omega^{\sharp}= \pm \omega$, we obtain $U=$ $\omega G_{1} T_{1} T_{2}^{-1} G_{2}^{-1} \in \Lambda G_{\sigma}$. Therefore, $L_{1}$ and $L_{2}$ are simultaneously realizable by the dressing $S=\omega G_{1} T_{1}=U G_{2} T_{2}$.

COROllary 3.7. Let

$$
L_{1}=\left(\begin{array}{cc}
v_{1} & 0 \\
p & v_{1}^{-1}
\end{array}\right), L_{2}=\left(\begin{array}{cc}
v_{2} & q \\
0 & v_{2}^{-1}
\end{array}\right) \in \Lambda G_{\sigma}^{C} .
$$

Assume that $v_{j}^{b}=v_{j}^{-1}, v_{j}, p$ and $q$ are holomorphic around $S^{1}$ and none of $v_{j}-v_{j}^{-1}$, $p$ and $q$ is identically zero on $S^{1}$. Then $L_{1}$ and $L_{2}$ are simultaneously realizable if the following three conditions hold:

(1) $\frac{p}{v_{1}^{-1}-v_{1}}, \frac{-q}{v_{2}^{-1}-v_{2}}$ are finite on $S^{1}$;

(2) $\frac{p q}{\left(v_{1}^{-1}-v_{1}\right)\left(v_{2}^{-1}-v_{2}\right)}+1= \pm f f^{b}$ for some $f \in \mathcal{W}^{+}$which is finite, nonvanishing on $S^{1}$ and nonvanishing holomorphic on $D$;

(3) $-i \frac{p}{q^{b}}\left(\frac{v_{2}^{-1}-v_{2}}{v_{1}^{-1}-v_{1}}\right)= \pm g g^{b}$ for some $g \in \mathcal{W}^{+}$which is finite, nonvanishing on $S^{1}$ and nonvanishing holomorphic on D. Here the sign is chosen in same order as in (2).

PROOF. From Lemma 3.3 and Remark 3.4, the lower and upper triangular loops are realizable if $p /\left(v_{1}^{-1}-v_{1}\right)$ and $-q /\left(v_{2}^{-1}-v_{2}\right)$ are finite on $S^{1}$, which is satisfied by 
the first condition. Set

$$
T_{1}=\left(\begin{array}{cc}
1 & 0 \\
\frac{p}{v_{1}^{-1}-v_{1}} & 1
\end{array}\right) \quad \text { and } \quad T_{2}=\left(\begin{array}{cc}
1 & \frac{-q}{v_{2}^{-1}-v_{2}} \\
0 & 1
\end{array}\right) .
$$

It is clear that $T_{1}, T_{2} \in \Lambda G_{\sigma}^{C}$ and

$$
T_{j} L_{j} T_{j}^{-1}=\left(\begin{array}{cc}
v_{j} & 0 \\
0 & v_{j}^{-1}
\end{array}\right)
$$

A straightforward computation shows that

$$
T_{1} T_{2}^{-1}=\left(\begin{array}{cc}
1 & \frac{q}{v_{2}^{-1}-v_{2}} \\
\frac{p}{v_{1}^{-1}-v_{1}} & \frac{p q}{\left(v_{1}^{-1}-v_{1}\right)\left(v_{2}^{-1}-v_{2}\right)}+1
\end{array}\right) .
$$

By Theorem 3.6, $L_{1}$ and $L_{2}$ are simultaneously realizable if and only if the second and third conditions in the corollary are satisfied.

REMARK 3.8. Condition (3) in Corollary 3.7 can be rephrased as

$$
-i \frac{p}{q^{b}}\left(\frac{v_{2}^{-1}-v_{2}}{v_{1}^{-1}-v_{1}}\right)=\frac{-p q}{\left(v_{1}^{-1}-v_{1}\right)\left(v_{2}^{-1}-v_{2}\right)} \cdot \frac{\left(v_{2}^{-1}-v_{2}\right)\left(v_{2}^{-1}-v_{2}\right)^{b}}{i q q^{b}} .
$$

Since $q$ is an odd function, $\left(i q q^{b}\right)^{b}=i q q^{b}$ holds. Then using Lemma 3.5,

$$
\frac{\left(v_{2}^{-1}-v_{2}\right)\left(v_{2}^{-1}-v_{2}\right)^{b}}{i q q^{b}}= \pm l l^{b}
$$

for some $l \in \mathcal{W}^{+}$which is finite, nonvanishing on $S^{1}$ and nonvanishing holomorphic on $D$. Therefore, the third condition in Corollary 3.7 can be replaced by:

(3) $\frac{ \pm p q}{\left(v_{1}^{-1}-v_{1}\right)\left(v_{2}^{-1}-v_{2}\right)}= \pm g g^{b}$ for some $g \in \mathcal{W}^{+}$which is finite, non-vanishing on $S^{1}$ and non-vanishing holomorphic on $D$.

Here, the sign is determined from (3.3).

\section{Totally symmetric surfaces}

In this section, we show the existence of the totally symmetric CMC surfaces in $\boldsymbol{H}^{3}$ with mean curvature $0 \leq H<1$.

THEOREM 4.1. Let $\eta$ be the following holomorphic potential:

$$
\eta=-\left(\begin{array}{cc}
0 & \lambda^{-1} \\
\lambda X^{2} & 0
\end{array}\right) \frac{d z}{z}+\left(\begin{array}{cc}
0 & \lambda^{-1} \\
\lambda X^{2} & 0
\end{array}\right) \frac{d z}{z-1}+\left(\begin{array}{cc}
0 & 0 \\
\lambda\left(X^{2}-1 / 4\right) & 0
\end{array}\right) d z
$$


where $a, b \in \boldsymbol{R}, X=\sqrt{b^{2}-a^{2}+a b\left(\lambda^{2}-\lambda^{-2}\right)}$ with $b^{2}-a^{2}+a b\left(e^{q}-e^{-q}\right)=1 / 4$, $q \in \boldsymbol{R}_{\geqq 0}$. Assume that

$$
b^{2} \not \equiv a^{2} \bmod S \quad \text { and } \quad-1+4 \sin ^{2}(\pi X) \neq 0 \quad \text { on } \lambda \in S^{1},
$$

where $S=\left\{y \in N \mid x^{2}=y\right.$ for some $\left.x \in Z\right\}$. Then there exists an immersion, possibly singular, from $\hat{\boldsymbol{C}} \backslash\{0,1, \infty\}$ into $\boldsymbol{H}^{3}$ with constant mean curvature $0 \leq H=\tanh q<1$ via the generalized Weierstrass type representation.

PROOF. Set

$$
\eta=\left(\begin{array}{cc}
0 & \eta_{12} \\
\eta_{21} & 0
\end{array}\right) d z \quad \text { and } \quad C=\left(\begin{array}{cc}
y_{1}^{\prime} / \eta_{12} & y_{1} \\
y_{2}^{\prime} / \eta_{12} & y_{2}
\end{array}\right)
$$

where $y_{1}$ and $y_{2}$ are the fundamental solutions of

$$
y^{\prime \prime}-\frac{\eta_{12}^{\prime}}{\eta_{12}} y^{\prime}-\eta_{12} \eta_{21} y=0 .
$$

Then it is easy to see that $C$ satisfies $d C=C \eta$. Since

$$
\eta_{12}=-\frac{\lambda^{-1}}{z}+\frac{\lambda^{-1}}{z-1} \text { and } \eta_{21}=\frac{-\lambda X^{2}}{z}+\frac{\lambda X^{2}}{z-1}+\lambda\left(X^{2}-1 / 4\right),
$$

we compute

$$
-\frac{\eta_{12}^{\prime}}{\eta_{12}}=\frac{1}{z}+\frac{1}{z-1}
$$

and

$$
-\eta_{12} \eta_{21}=-\frac{X^{2}}{z^{2}}-\frac{X^{2}}{(z-1)^{2}}+\left(\frac{1}{4}+X^{2}\right)\left(-\frac{1}{z}+\frac{1}{z-1}\right) .
$$

Setting $z_{1}=0, z_{2}=1, z_{3}=\infty, a_{1}=a_{2}=1, a_{3}=0, b_{1}=b_{2}=-X^{2}, b_{3}=1 / 4-X^{2}$ and $c_{2}=-c_{1}=1 / 4+X^{2}$,

$$
-\frac{\eta_{12}^{\prime}}{\eta_{12}}=\sum_{j=1}^{2} \frac{a_{j}}{z-z_{j}}, \quad-\eta_{12} \eta_{21}=\sum_{j=1}^{2}\left\{\frac{b_{j}}{\left(z-z_{j}\right)^{2}}+\frac{c_{j}}{z-z_{j}}\right\}
$$

and

$$
\sum_{j=1}^{2} c_{j}=0, \quad \sum_{j=1}^{3} a_{j}=2, \quad b_{3}=\sum_{j=1}^{2}\left(b_{j}+c_{j} z_{j}\right)
$$

These are equations given in [5, Section 3.3]. It is easy to see that (4.3) is equivalent to the Gauss hypergeometric equation. Let $\gamma_{1}, \gamma_{2}$ and $\gamma_{3}$ be the simple closed loops around $z=z_{1}, z_{2}$ and $z_{3}$ from some base point $z_{*}$, respectively. Moreover, let $M_{1}, M_{2}$ and $M_{3}$ be monodromy matrices corresponding to $\gamma_{1}, \gamma_{2}$ and $\gamma_{3}$, respectively, that is, $\gamma_{j}^{*} C=M_{j} C(j=1,2,3)$. It is well known that

$$
M_{1} M_{2} M_{3}=\mathrm{id},
$$


since $\gamma_{1} \gamma_{2} \gamma_{3}=1$. Following the discussion in [5, Section 3.7], the monodromy matrices for a suitable fundamental system of (4.3) can be computed explicitly as

$$
\begin{gathered}
M_{1}=\left(\begin{array}{cc}
e^{2 \pi i r_{1,+}} & 0 \\
\left(e^{2 \pi i r_{1,+}}-e^{2 \pi i r_{1,-}}\right) d_{1} \lambda^{-1} & e^{2 \pi i r_{1,-}}
\end{array}\right), \\
M_{2}=\left(\begin{array}{cc}
e^{2 \pi i r_{2,-}} & \left(e^{2 \pi i r_{2,+}}-e^{2 \pi i r_{2,-}}\right) d_{2} \lambda \\
0 & e^{2 \pi i r_{2,+}}
\end{array}\right),
\end{gathered}
$$

where

$$
r_{j, \pm}=\frac{1}{2}\left\{1-a_{j} \pm \sqrt{\left(1-a_{j}\right)^{2}-4 b_{j}}\right\}
$$

and

with

$$
d_{1}=\frac{\Gamma\left(1-\delta_{1}\right) \Gamma\left(\delta_{2}\right)}{\Gamma\left(\delta_{2}-\alpha\right) \Gamma\left(\delta_{2}-\beta\right)}, \quad d_{2}=\frac{\Gamma\left(1-\delta_{2}\right) \Gamma\left(\delta_{1}\right)}{\Gamma\left(\delta_{1}-\alpha\right) \Gamma\left(\delta_{1}-\beta\right)},
$$

$$
\begin{aligned}
\delta_{1} & =1+\sqrt{\left(1-a_{1}\right)^{2}-4 b_{1}}=1+2 X, \quad \delta_{2}=1+\sqrt{\left(1-a_{2}\right)^{2}-4 b_{2}}=1+2 X, \\
\alpha & =\frac{1}{2}\left\{\delta_{1}+\sqrt{\left(1-a_{2}\right)^{2}-4 b_{2}}+\sqrt{\left(1-a_{3}\right)^{2}-4 b_{3}}\right\}=\frac{1}{2}(1+6 X), \\
\beta & =\frac{1}{2}\left\{\delta_{1}+\sqrt{\left(1-a_{2}\right)^{2}-4 b_{2}}-\sqrt{\left(1-a_{3}\right)^{2}-4 b_{3}}\right\}=\frac{1}{2}(1+2 X) .
\end{aligned}
$$

Since $\delta_{1}=\delta_{2}$ and $r_{1, \pm}=r_{2, \pm}$,

$$
d=d_{1}=d_{2}=\frac{-1}{2 \sin \pi X}, \quad e^{2 \pi i r_{ \pm}}=e^{2 \pi i r_{1, \pm}}=e^{2 \pi i r_{2, \pm}} .
$$

The first condition in Corollary 3.7 can be computed as

$$
\frac{p}{v_{1}^{-1}-v_{1}}=-d \lambda^{-1}, \quad \frac{-q}{v_{2}^{-1}-v_{2}}=-d \lambda .
$$

Since $d$ is finite on $S^{1}$ by the assumption, the first condition is satisfied.

The third condition in Corollary 3.7 can be computed as

$$
-i \frac{p}{q^{b}}\left(\frac{v_{2}^{-1}-v_{2}}{v_{1}^{-1}-v_{1}}\right)=1
$$

Thus the third condition is clearly satisfied.

Since $X^{b}=X$ (see the proof of Lemma 2.6), we see that $d^{b}=d$. A direct computation shows that the second condition can be rephrased as

$$
\frac{p q}{\left(v_{1}^{-1}-v_{1}\right)\left(v_{2}^{-1}-v_{2}\right)}+1=-d^{2}+1=\frac{-1+4 \sin ^{2} \pi X}{4 \sin ^{2} \pi X} .
$$

From the assumption, the denominator and numerator of (4.4) never vanish on $S^{1}$. Therefore, one can rephrase (4.4) as

$$
-d^{2}+1=h^{2} \quad \text { with } h=\frac{\sqrt{-1+4 \sin ^{2} \pi X}}{2 \sin \pi X} .
$$


Since $h$ is a finite, nonvanishing function on $S^{1}$, using the Birkhoff decomposition in Lemma 3.5 for $h$, we have $h=\epsilon k_{+} k_{+}^{b}$, where $\epsilon \in\{ \pm 1\}$ and $k_{+}$is an element in $\mathcal{W}^{+}$ which is finite and nonvanishing on $S^{1}$ and nonvanishing holomorphic on $D$. Thus

$$
-d^{2}+1=h^{2}=k_{+}^{2} k_{+}^{b 2}
$$

Finally, setting $f=k_{+}^{2}$, we have $-d^{2}+1=f f^{b}$. Thus the second condition is satisfied, and from Corollary 3.7, $M_{1}$ and $M_{2}$ can be simultaneously realizable via some dressing $S \in \Lambda G_{\sigma}^{C}$.

Let $S \in \Lambda G_{\sigma}^{C}$ be the dressing which realizes $M_{1}$ and $M_{2}$ simultaneously. It is easy to see that $M_{3}$ is also realizable by $S$, since $M_{1} M_{2} M_{3}=$ id. Then from Theorem 3.2, $S M_{j} S^{-1} \in \Lambda G_{\sigma}(j=1,2)$ can be computed as

$$
S M_{j} S^{-1}=U_{j} G_{j+}\left(\begin{array}{cc}
e^{2 \pi i r_{+}} & 0 \\
0 & e^{2 \pi i r_{-}}
\end{array}\right) G_{j+}^{-1} U_{j}^{-1},
$$

where $U_{j} \in \Lambda G_{\sigma}$ and $G_{j+} \in \Lambda^{+} G_{\sigma}^{C}$. From the assumption, $\left.S M_{j} S^{-1}\right|_{\lambda=e^{-q / 2}}=-\mathrm{id}$, $(j=1,2)$ and $\left.S M_{3} S^{-1}\right|_{\lambda=e^{-q / 2}}=$ id. Therefore, from Lemma $2.5, \gamma_{1}, \gamma_{2}$ and $\gamma_{3}$ are elements of the fundamental group of the resulting CMC immersion $\psi$. This completes the proof.

\section{REMARK 4.2.}

(1) It is known that CMC immersions from $\hat{\boldsymbol{C}} \backslash\{0,1, \infty\}$ into $\boldsymbol{R}^{3}$ can be constructed from potentials $\eta$ of the form (4.1). However, in this case $a$ and $b$ need to be chosen differently; see [5, 11].

(2) It could be possible to construct nontotally symmetric trinoids from potentials $\eta$ of the form (4.1) by choosing the different $X$ in the coefficient matrix of $d z / z, d z /(1-z)$ and $d z$, respectively.

(3) Since the Iwasawa decomposition Theorem 2.2 is not global in general, the CMC surfaces constructed by this method have singularities, where the surfaces tend to infinity. These singularities are different from the three regular singularities of the Gauss hypergeometric equation.

\section{References}

[1] J. Dorfmeister and G. Haak, 'Construction of non-simply connected CMC surfaces via dressing', J. Math. Soc. Japan 55(2) (2003), 335-364.

[2] J. Dorfmeister, J. Inoguchi and S.-P. Kobayashi, 'Constant mean curvature surfaces in hyperbolic 3-space via loop groups', Preprint, 2009.

[3] J. Dorfmeister and S.-P. Kobayashi, 'Coarse classification of constant mean curvature cylinders', Trans. Amer. Math. Soc. 359(6) (2007), 2483-2500 (electronic).

[4] J. Dorfmeister and H. Wu, 'Unitarization of loop group representations of fundamental groups', Nagoya Math. J. 187 (2007), 1-33.

[5] J. Dorfmeister and H. Wu, 'Construction of constant mean curvature $n$-noids from holomorphic potentials', Math. Z. 258(4) (2008), 773-803. 
[6] I. Gohberg, 'The factorization problem in normed rings, functions of isometric and symmetric operators, and singular integral equations', Russian Math. Surveys 19(1) (1964), 63-114.

[7] M. Kilian, S.-P. Kobayashi, W. Rossman and N. Schmitt, 'Constant mean curvature surfaces of any positive genus', J. Lond. Math. Soc. (2) 72(1) (2005), 258-272.

[8] M. Kilian, W. Rossman and N. Schmitt, 'Delaunay ends of constant mean curvature surfaces', Compositio Math. 144(1) (2008), 186-220.

[9] S.-P. Kobayashi, 'Real forms of complex surfaces of constant mean curvature', Trans. Amer. Math. Soc., to appear.

[10] S. Lang, Complex Analysis, 4th edn, Graduate Texts in Mathematics, 103 (Springer, New York, 1999).

[11] N. Schmitt, M. Kilian, S.-P. Kobayashi and W. Rossman, 'Unitarization of monodromy representations and constant mean curvature trinoids in 3-dimensional space forms', J. Lond. Math. Soc. (2) 75(2) (2007), 563-581.

SHIMPEI KOBAYASHI, Graduate School of Science and Technology,

Hirosaki University, Hirosaki, 036-8561, Japan

e-mail: shimpei@cc.hirosaki-u.ac.jp 